\title{
Effects of atropine on autonomic indices based on electrocardiographic R-R intervals in healthy volunteers
}

\author{
P O O Julu, R G Hondo
}

\begin{abstract}
The dose dependent responses to atropine of 11 indices of autonomic function were investigated in 10 healthy volunteers. Five subjects were given cumulative doses of atropine $(0 \cdot 1-2 \cdot 0$ $\mathrm{mg} / 70 \mathrm{~g}$, intravenously). The other five received equivalent volumes of water for injection. The ratio of the longest to the shortest $\mathbf{R}-\mathbf{R}$ intervals in the electrocardiogram during forced expirations and deep inspirations (respiratory ratio), deviations in the periods of consecutive cardiac cycles, the ratio of the longest to the shortest $R-R$ intervals during a Valsalva manoeuvre (Valsalva ratio), the ratio of the 30 th to the 15 th $R-R$ intervals after standing up from a low sitting position (30:15 ratio), and the means of $R-R$ intervals recorded in standing or lying positions were calculated with different computer algorithms. Sympathetic orthostatic effects on the above indices were measured by comparing values in supine and standing positions after atropinisation. The recumbent respiratory ratio was the most sensitive test to atropinisation. Its maximum response was a $97 \%$ decrease, indicating specificity for the vagal tone. The decrease in other indices in response to atropine ranged from $34 \%$ to $94 \%$. The average orthostatic effect on the indices after atropinisation was a $14.6 \%$ increase. The Valsalva ratio failed to respond significantly to any degree of muscarinic antagonism. In conclusion, the Valsalva ratio is unlikely to be closely related to the parasympathetic control of the heart. However, the resting vagal tone can be selectively measured in a live and conscious person by using the respiratory ratio.
\end{abstract}

Department of Physiology, University of Zimbabwe, Harare, Zimbabwe P O O Julu

R G Hondo

Correspondence to:

Dr POO Julu

Clinical Neurophysiology,

INS Southern General

Hospital, Glasgow G51 4TF,

UK

Received 4 February 1991 and in revised form

30 April 1991.

Accepted 3 May 199
Cardiac denervation may be one important cause of sudden death in diabetes mellitus. ${ }^{1-3}$ For this reason non-invasive techniques are needed to assess the degree of autonomic control of the heart in diabetes. ${ }^{4}$ One of the techniques commonly used is to quantify the variation in duration of cardiac cycles. Individual periods are reflexly adjusted beat by beat, creating a variation that depends on the degree of nervous control exerted on the heart. These variations are quantified either as the standard deviation of many $R-R$ intervals in an electroencephalogram (ECG), ${ }^{4}$ or as changes in heart rate during special manoeuvres-for example, the Valsalva manoeuvre $^{5}$ or rising from a recumbent position. ${ }^{6}$ Periods of cardiac cycles also vary during inspirations and expirations (respiratory sinus arrhythmia). ${ }^{7}$ The amplitude of this variation is related to the level of parasympathetic control of the heart. ${ }^{8}$ This variation can be quantified as a ratio of $R-R$ intervals during deep breathing exercises. ${ }^{9}$ These tests provide indices of autonomic function.

According to the above indices, the nervous control of the heart in patients with diabetic autonomic neuropathy is reduced. ${ }^{10}{ }^{11}$ However, the magnitude of abnormalities from the two branches of the autonomic system that can be measured in each test is not known.

The aim of this study was to plot the dose dependent responses to atropine, of the variations in $R-R$ intervals, measured in 11 commonly used tests, in healthy volunteers. The most sensitive test to atropinisation would thus be used to measure specifically the vagal tone in either patients or any other physiological studies on humans. The study elucidates the magnitude of changes attributable to the vagal tone in these indices.

\section{Methods}

Subjects

Ten volunteers aged between 20 and 42 were recruited with the approval of the local ethical committee. The procedures were explained to them before obtaining their written consent. Each experiment was started in the morning before the subject had breakfast. Fasting was maintained throughout the experiment. The subjects rested supine for five minutes at the beginning of each experiment, with lead I of the ECG connected. A preliminary measurement of $\mathbf{R}-\mathbf{R}$ intervals during this bed rest ensured that the subject was relaxed and the ECG was stable. The indices below were measured three times during the resting period using the procedures described by Mubagwa and Adler. ${ }^{12}$

(1) Mean R-R intervals (ms) of 250 consecutive cycles were recorded in supine and standing positions and the ratio of the mean values supine to those standing were calculated.

(2) Deviations in 250 consecutive $R-R$ intervals (ms) were assessed from the SDs of the $R-R$ intervals in supine and standing positions and from the mean of the absolute values 
of the beat to beat differences between $R-R$ intervals in supine and standing positions.

(3) Ratios of $\mathbf{R}-\mathbf{R}$ intervals during special manoeuvres were calculated: (a) Valsalva ratio, the ratio of the longest to the shortest $R-R$ intervals during a Valsalva manoeuvre lasting for $15 \mathrm{~s} ;(b)$ the 30:15 ratio, the ratio of the 30th to the 15th $R-R$ intervals immediately after standing up from a low sitting position; and (c) the respiratory ratio, the ratio of the longest to the shortest $\mathbf{R}-\mathbf{R}$ intervals during three cycles of forced expirations and deep inspirations. Each cycle lasted for $10 \mathrm{~s}$, and was recorded in supine and standing positions.

\section{Drug treatment}

Five subjects chosen at random were given atropine sulphate (Central African Pharmaceutical Society, Harare, Zimbabwe) intravenously in seven separate doses of $0.1,0.1,0.2$, $0.2,0.4,0.5$ and $0.5 \mathrm{mg}$ per $70 \mathrm{~kg}$. These doses were achieved by diluting $0.6 \mathrm{mg}$ atropine in standard ampoules so that $0.1 \mathrm{ml}$ was equivalent to $0 \cdot 1 \mathrm{mg}$ of atropine. A tuberculin $1 \mathrm{ml}$ syringe was then used to administer the drug. The other five subjects were given equivalent volumes of water for injection. All the tests above were done starting five minutes after each intravenous injection. This was enough time lag for the heart rate to stabilise after injections. The injections built up cumulative doses of atropine starting from $0 \cdot 1$, then $0 \cdot 2,0.4,0.6,1.0,1 \cdot 5$, and $2 \cdot 0 \mathrm{mg}$ per $70 \mathrm{~kg}$ in each subject. The individual experiments lasted between four and nine hours.

Details of the test procedures and the equipment used in this study are as described by Mubagwa and Adler. ${ }^{12}$ The values obtained from tests done after each dose of atropine or water were later expressed as percentages of the values obtained during the resting period. These percentages were used to plot the dose dependent response curves of changes caused by atropine or water.

\section{Statistical analysis}

Values obtained from each test were expressed as means, and when appropriate SD and SE were calculated. Two tailed $t$ tests were used to compare mean values obtained after each dose of atropine to equivalent control values. The confidence limit for significance was $95 \%$ $(p<0.05)$. The coefficients of variation in each index experiment were calculated with values obtained from control subjects.

\section{Results \\ Ratios of $R-R$ intervals during special manoeuvres}

Respiratory ratio recorded in the supine position (RRL) was the most sensitive test in the whole study. The range was between 1.16 and 1.66 (mean 1.37 (SE 0.05)) during the resting period (all volunteers). RRL was between 1.01 and $1.02(1.012(0.002)$, after atropinisation $(n=5)$. This was a $97 \%$ decrease in response to atropine(figure 1). Coefficients of variation were between $2 \%$ and $5 \%(3.0 \%(0.5 \%))$, in control subjects $(n=5)$. There was no increase in RRL above the initial value in response to low doses of atropine (figure 1). Recordings made in standing positions (RRS) were between 1.19 and $1.70(1.35(0.05))$ during the resting period. RRS was between 1.04 and $1.05(1.048(0.002))$ after atropinisation. This was an $88 \%$ decrease in response to atropine (figure 1). The $9 \%$ difference between the maximum decrease in RRL and RRS was significant $(p<0.001)$. Coefficients of variation of RRS were between $2 \%$ and $5 \%(3.4 \%(0.6 \%))$. There was no increase in RRS in response to low doses of atropine (figure 1 ).

The values of the 30 to 15 ratio were between 1.11 and $1.28(1.20(0.02))$ during the resting period. They were between 1.04 and 1.05 $(1.045(0.003))$ after atropinisation. This was a $78 \%$ drop in response to atropine (figure 1). Coefficients of variation in the control subjects were between $2 \%$ and $8 \%(4 \cdot 3 \%(1.0 \%))$. There was a significant $64 \%$ initial increase $(p<0.01)$ in response to a very low dose of atropine (figure 1 ).

The Valsalva ratios were between 1.38 and $2.33(1.82(0.08))$ during the resting period. They were between 1.01 and $1.68(1.41(0.12))$ after atropinisation. All changes were not significant (figure 1). Coefficients of variation were between $4 \%$ and $9 \%(6 \cdot 2 \%(1 \cdot 3 \%))$.

\section{Deviations of consecutive $R-R$ intervals}

The mean of absolute values of the beat to beat variations (BV) recorded in the supine position (BVL) was the most sensitive test in this category. The range of BVL was between 15 and $63 \mathrm{~ms}(37.9(5.1) \mathrm{ms})$ during the resting period. It was between 2 and $3 \mathrm{~ms}(2 \cdot 6(0 \cdot 2) \mathrm{ms})$ after atropinisation. This was a $94 \%$ decrease in response to atropine (figure 2). Coefficients of variation were between $10 \%$ and $19 \%(13.5 \%$ $(1 \cdot 7 \%))$. If recorded in a standing position (BVS) the values were between 6 and $18 \mathrm{~ms}$ $(12.3(1 \cdot 2) \mathrm{ms})$ during the resting period. BVS was between 2 and $4 \mathrm{~ms}(3.0(0.3) \mathrm{ms})$ after atropinisation. This was a $77 \%$ drop in response to atropine (figure 2 ). The $17 \%$ difference between the maximum drop in BVL and BVS was significant $(p<0.001)$. Coefficients of variation of BVS were between $8 \%$ and $12 \%$ $(10.3 \%(1.0 \%))$

The SD of R-R intervals recorded in supine positions (L-SD) was between 28 and $62 \mathrm{~ms}$ $(47 \cdot 5(3 \cdot 7) \mathrm{ms})$ during the resting period. L-SD was between 2 and $7 \mathrm{~ms}(4.8(1.0) \mathrm{ms})$ after atropinisation. It decreased by $90 \%$ in response to atropine (figure 2). Coefficients of variation were between $9 \%$ and $20 \%(14.7 \%$ $(2 \cdot 8 \%))$. The initial increase of L-SD above the starting value in response to a very low dose of atropine was not significant (figure 2). If recorded in a standing position (S-SD) it was between 19 and $39 \mathrm{~ms}(30.7(2.3) \mathrm{ms})$ during the resting period. S-SD was between 4 and 13 $(8 \cdot 2(1 \cdot 7))$ after atropinisation. It decreased by $75 \%$ (figure 2 ) in response to atropine. The $15 \%$ difference between the maximum decrease in L-SD and S-SD was significant $(p<0.05)$. Coefficients of variation of S-SD were between $11 \%$ and $29 \%(18 \cdot 4 \%(3 \cdot 2 \%))$. There was a significant $37 \%$ increase in S-SD $(p<0.001)$ 
RRL

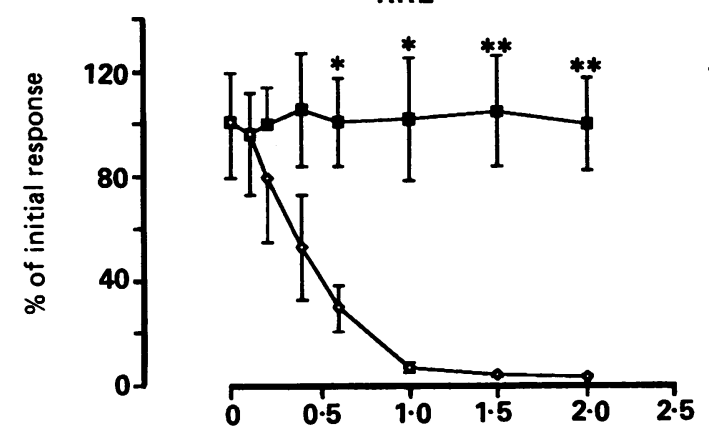

$30: 15$ ratio

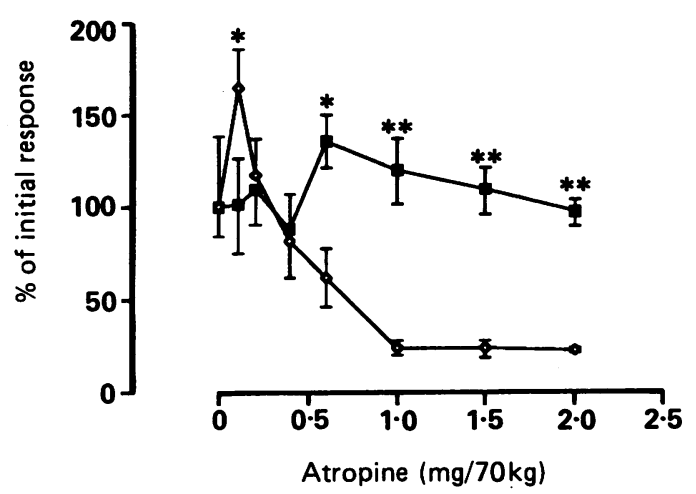

RRS
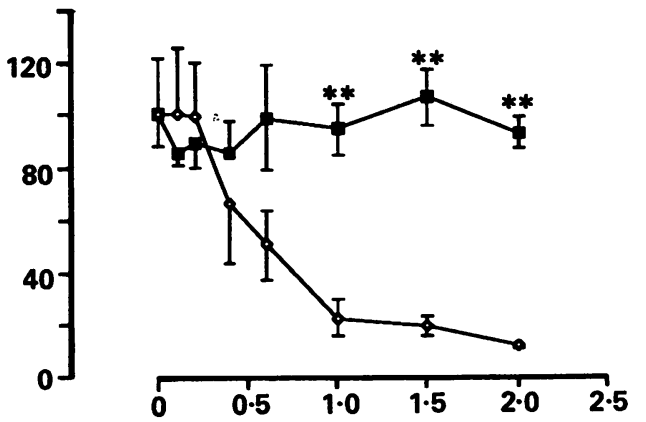

Valsalva ratio

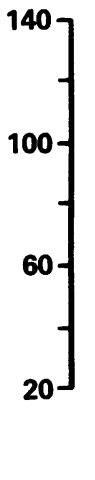

Figure 1 Dose dependent response to atropine of the ratios of electrocardiographic $R-R$ intervals during special manoeuvres. $R R L=$ ratios of the longest to the shortest $R-R$ intervals during deep breathing exercises in supine positions. $R R S=$ the same ratios recorded in standing positions. 30:15Ratio = the ratio of the 30th to the 15th intervals immediately after standing up from a low sitting position: Valsalva ratio = the ratio of the longest to the shortest $R-R$ intervals during a Valsalva manoeuvre lasting for $15 \mathrm{~s}$. (Subjects given atropine ( $\diamond$ ), and those given equivalent volumes of water for injection (D). Vertical bars are $S E S(n=5)$.) The significance of the differences between values obtained after various doses of atropine and water were tested using two tailed $t$ tests, where ${ }^{\star} p<0.01$ and ${ }^{\star \star} p<0.001$. Mean values of RRL and RRS did not vary much throughout the 4 to 9 hours of the experiments in control subjects. Note that 30:15 ratio increased in response to a small dose of atropine and Valsalva ratio did not respond significantly to any dose of atropine.

above the starting value in response to the first small dose of atropine (figure 2). This was followed by an additional $36 \%$ increase $(p<0.01)$ in response to a second small dose.

\section{Mean $R-R$ intervals}

In the supine position the range of means of $\mathbf{R}-\mathbf{R}$ intervals recorded in individual volunteers was $876-1340 \mathrm{~ms}(1026.6(48.9) \mathrm{ms})$ during the resting period. It was $490-628 \mathrm{~ms}(561 \cdot 8(28 \cdot 7)$ ms) after atropinisation. The means decreased by $46 \%$ in response to atropine (figure 3 ). Coefficients of variation were between $2 \%$ and $7 \%(3.6 \%(0.9 \%))$. The means in each volunteer when standing were between 617 and 1023 ms (748.9 (43.2) ms) during the resting period and between 439 and $599 \mathrm{~ms}(496 \cdot 4(27 \cdot 1) \mathrm{ms})$ after atropinisation. This set of means decreased by only $34 \%$, the lowest response to atropine in the whole study (figure 3). Coefficients of variation were between $2 \%$ and $6 \%$ $(4 \cdot 1 \%(0.6 \%))$. The $12 \%$ difference between the maximum decrease in the values recorded in supine and standing positions was significant ( $\mathrm{p}<0.05)$.

Ratios of the mean R-R intervals recorded in supine to those in standing positions (LS-ratio) were between 1.21 and $1.54(1.38(0.05))$ during the resting period. They were between 1.00 and
$1.30(1.15(0.06))$ after atropinisation. The ratios decreased by $60 \%$ in response to atropine (figure 3). Coefficients of variation were between $3 \%$ and $5 \%$ during the experiment $(3.9 \%$ $(0.5 \%))$. The apparent initial increase of LS-ratio was not significant (figure 3).

\section{Discussion}

A clinical index that is closely linked with the parasympathetic control of the heart will respond proportionally to muscarinic antagonism. Graded doses of atropine provide varying degrees of muscarinic antagonism. This can be used to identify the responses of clinical indices that are dose-dependent and therefore genuine responses to muscarinic antagonism (figures 13). This procedure is different from the atropine test, in which heart rate is measured before and after a single large dose of atropine. RRL was sensitive to atropine and an accurate indicator of the vagal tone too. The doses of atropine at which RRL was maximally reduced compares well with those which caused maximum haemodynamic ${ }^{13}$ and heart rate changes ${ }^{14}$ in healthy volunteers. RRL was reproducible for most of the daytime. Its coefficient of variation over a period of up to nine hours was only $3.0 \%$ $(0.5 \%)$. RRL could be useful as a single clinical 

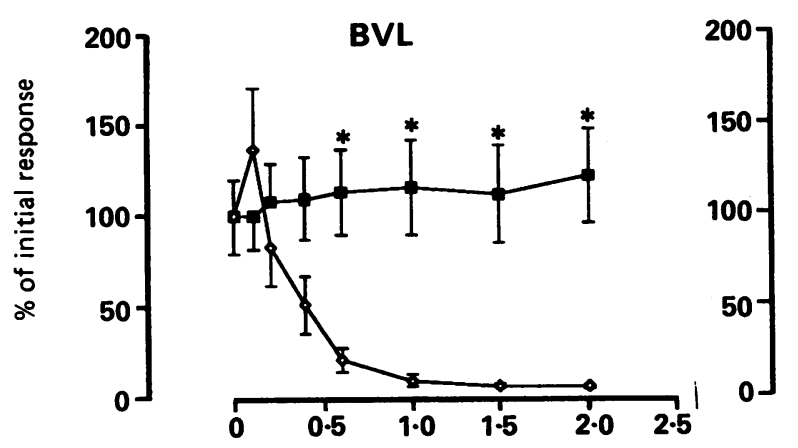

BVS
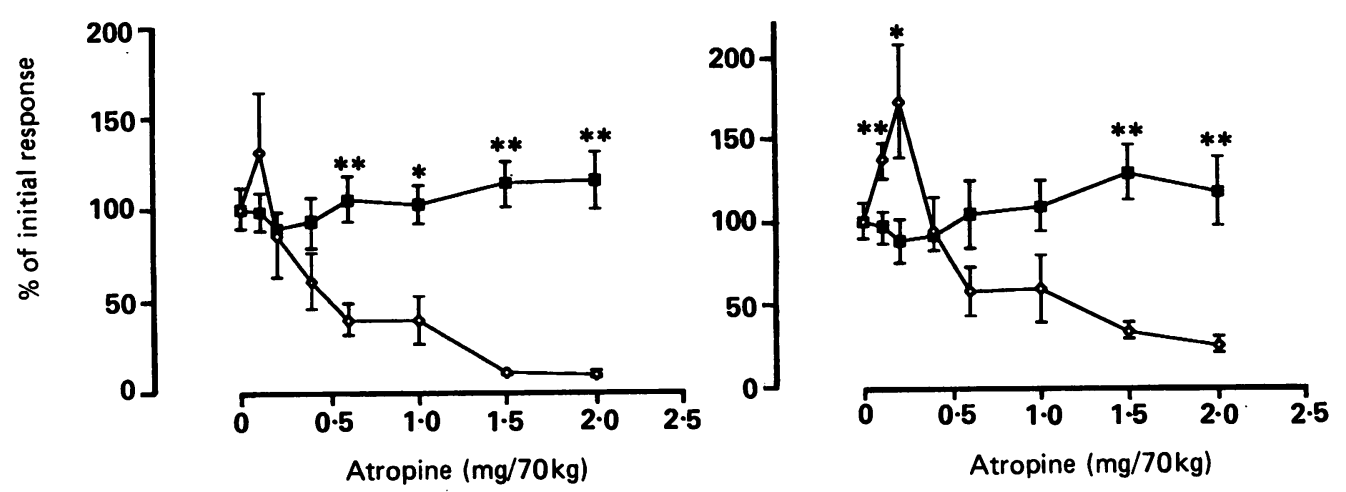

Figure 2 Dose dependent response to atropine of the deviations measured in consecutive $R-R$ intervals of the ECG. $B V L=$ the mean of the absolute difference between consecutive $R-R$ intervals recorded in a supine position. $B V S=$ the same difference recorded in a standing position. $L-S D$ and $S-S D$ are $S D s$ of $R-R$ intervals recorded in supine and standing positions respectively. (Subjects given atropine $(\diamond)$, and those given equivalent volumes of water for injection (D). Vertical bars are SEs $(n=5)$.) Mean values of all four indices did not vary much during the 4 to 9 hours of the experiments in control subjects. The significance of the differences between values obtained after various doses of atropine and water were tested using two tailed t tests, where $\star_{p}<0.01$, and $\star^{\star} p<0.001$. Only $S-S D$ increased significantly in response to very low doses of atropine.

index for selectively measuring vagal tone in patients. In addition, the dose dependent responses of RRL to atropine could be used to calculate a linear scale for vagal tone with the formula given by Katona and Jih. ${ }^{15}$ Such a scale will have an absolute zero representing no nerve impulses in the cardiac vagal nerve, ideal for comparing parasympathetic regulations of the heart in different groups of people.

We have elucidated the proportions of the vagal tone measurable by different tests (see results and figures $1-3$ ). They may be useful for the interpretation of these clinical tests. With power spectral analysis of heart rate, the sympathetic control of the heart was found to be negligible in a person resting in a supine position; it increased during sustained active standing. ${ }^{16}$ The average increase in the nervous control of the heart due to this sympathetic orthostatic regulation was $14.6 \%$ in our study. This average was derived from the $17 \%, 15 \%$, and $12 \%$ differences between lying and standing values of $S D, B V$, and mean $R-R$ intervals respectively at full atropinisation (see results). It may look as if the respiratory ratio (respiratory sinus arrhythmia) had no sympathetic component because it was $97 \%$ vagal in a supine position (figure 1). It was suggested in earlier studies that the sympathetic system does not contribute to respiratory sinus arrhythmia in the cat. ${ }^{17}$ But we found a $12 \%(p<0.001)$ non-vagal component when our volunteers were standing. The $9 \%$ difference between
RRL and RRS at full atropinisation was significant $(p<0.001)$. Neurograms recorded from the thoracic sympathetic trunk innervating the heart in anaesthetised cats showed phasic discharges, which only came during the expiratory phase of respiration. ${ }^{18}$ All these show a sympathetic component of the respiratory sinus arrhythmia. There is also a basal rhythmic variation in the periods of cardiac cycles after all nerve supplies have been cut. ${ }^{17} \mathrm{It}$ had little effect on RRL here but may contribute to the variations in the other tests.

The technique of choice for measuring the parasympathetic control of the heart would be by quantifying the variations in the periods of cardiac cycles as opposed to the average of the periods (see maximum responses to atropine in figures 1-3). Algorithms and manoeuvres can be optimised for measuring specific variations of the heart periods. SD measures the total variation (constant, slow, or fast) in a time space equal to the duration of data collection. It was 3 minutes or more in this study, depending on the heart rate. This is inbuilt in the statistical algorithm. BVL and BVS measure the fast variation that causes detectable differences between immediate consecutive heart periods. ${ }^{12}$ This variation is caused by the action of arterial baroreceptors on the sinoatrial node during the brief ejection period of each cardiac cycle. ${ }^{19}$ RRL and RRS measure the maximum variation of heart period synchronised with deep inspirations and forced expirations. This 
Figure 3 Dose dependent response to atropine of the means of $R-R$ intervals in the ECG. $L S$-ratio = the ratio of the means recorded in supine to that in standing positions. (Subjects given atropine $(\diamond)$, and those given equivalent volumes of water for injection (D). Vertical bars are SES $(n=5))$. The mean values recorded in supine position were constant throughout the 4 to 9 hours of the experiments in control subjects. The significance of the differences between values obtained after various doses of atropine and water were tested using two tailed $t$ tests, where $\star_{p}<$ 0.01 and $\star \star p<0.001$.
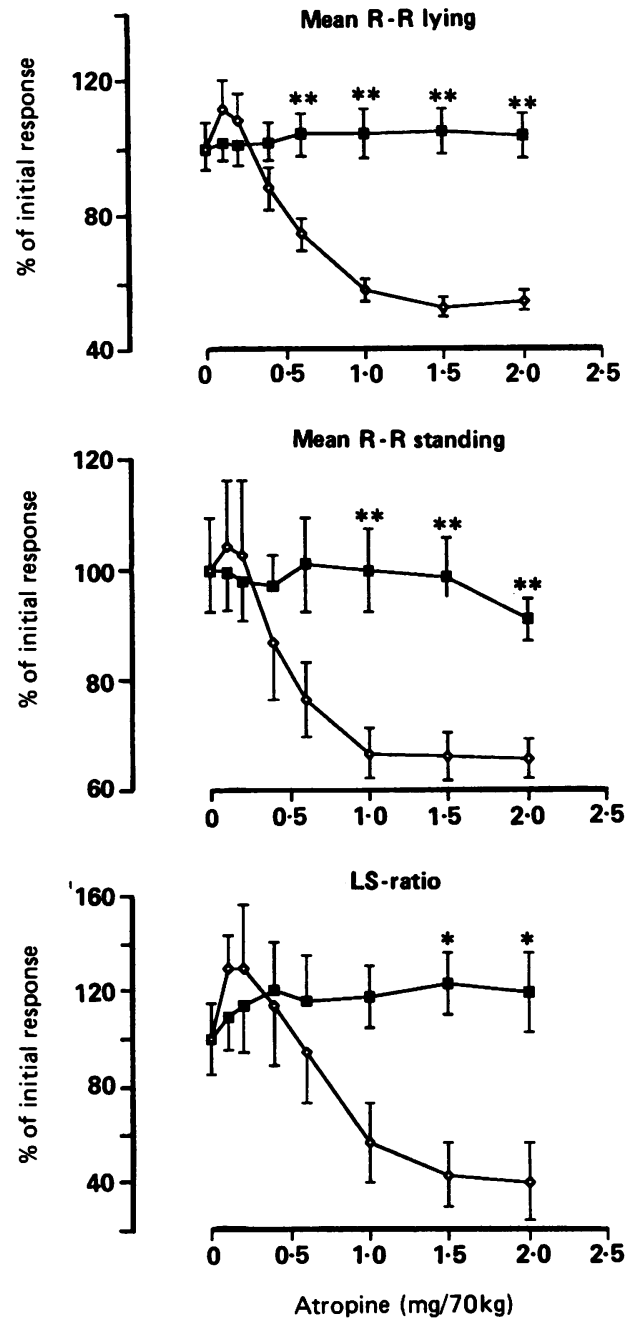

variation is caused by the modulation of excitability of vagal motor neurons in nucleus ambiguus by the inspiratory centre. ${ }^{20}$ The $30: 15$ ratio measures the immediate cardiac nervous response to active standing within $\mathbf{3 0}$ cardiac cycles. It was previously thought to be entirely vagal in origin. ${ }^{6}$ All these manoeuvres and algorithms had different efficiencies for measuring the parasympathetic control of the heart. The range was from $75 \%$ to $97 \%$ of the parasympathetic regulation (see results and figures 1-3).

One advantage of BVL is that no active participation by the patient is required to measure it. Being $94 \%$ responsive to atropine, it could be useful in anaesthesiology for measuring vagal tone in unconscious patients. Cardiorespiratory arrests due to abnormal cardiovascular reflexes are common in diabetic patients during anaesthesia. ${ }^{21}$ BVL could be used to assess the vagal tone in diabetic patients during anaesthesia. It was also interesting that only two indices, the $30: 15$ ratio and S-SD, increased significantly in response to low doses of atropine. Low doses of atropine are thought to excite the central nervous system, including the vagus nerve. ${ }^{22}$ RRL, BVL, and other indices that had high sensitivities to atropine did not increase significantly in response to low doses of atropine, as was expected.

The Valsalva ratio was not significantly affected by any degree of muscarinic antagon- ism produced by atropine. It also failed to significantly respond to the muscarinic antagonism of chloroquine in healthy volunteers. ${ }^{12}$ The Valsalva ratio is unlikely to be closely related to the parasympathetic control of the heart. This needs to be remembered because the Valsalva ratio is a popular test in diabetology. ${ }^{51123}$

In conclusion, $R R L$ was a specific test for the vagal tone on the heart. It could be useful for selective measurement of vagal tone in humans. The degree of parasympathetic regulation measured by other tests was variable and lower. The Valsalva ratio was probably not closely related to the parasympathetic control of the heart, and caution should be taken when interpreting this index in diabetology.

This study was supported by the Research Board, University of Zimbabwe, grant No 3131 1987, given to POO Julu. We are grateful to Scotia Pharmaceuticals for financing the technical assistance.

1 Wheeler T, Watkins PJ. Cardiac denervation in diabetes. BMJ 1973;4:584-6.

2 Lloyd-Mostyn RH, Watkins PJ. Defective innervation of heart in diabetic autonomic neuropathy. $B M J$ 1975;iii: 15-7.

3 Page MMcB, Watkins PH. The heart in diabetes: autonomic neuropathy and cardiomyopathy. Clinic Endocrinol Metab 1977;6:377-88

4 Bennett T, Farquhar IK, Hosking DJ, Hampton JR. Assessment of methods for estimating autonomic nervous control of the heart in patients with diabetes mellitus. Diabetes 1978;27:1167-74.

5 Sharpey-Schafer EP, Taylor PJ. Absent circulatory reflexes in diabetic neuritis. Lancet 1960; i:559-62.

6 Ewing DJ, Campbell IW, Murray A, Neilson JMM, Clarke BF. Immediate heart-rate response to standing: simple test for autonomic neuropathy in diabetes. $B M J$ 1978; i: $145-7$.

7 Anrep GV, Pascual W, Rossler R. Respiratory variations of the heart rate. I. The reflex mechanism of the respiratory arrhythmia. Proc R Soc Lond [Biol] 1936;119:191-217.

8 Fouad FM, Tarazi RC, Ferrario CM, Fighaly S, Alicandri C. Assessment of parasympathetic control of heart rate by a noninvasive method. Am J Physiol 1984;246:H838-2.

9 Hilsted J, Jensen SB. A simple test for autonomic neuropathy in juvenile diabetics. Acta Med Scand 1979;205:385-7.

10 Clarke BF, Ewing DJ, Campbell IW. Diabetic autonomic neuropathy. Diabetologia 1979;17:195-212.

11 Ewing DJ, Clarke BF. Diabetic autonomic neuropathy: present insights and future prospects. Diabetes Care 1986;9:648-65.

12 Mubagwa $\mathrm{K}$, Adler J. Muscarinic antagonist action of clinical doses of chloroquine in healthy volunteers. J Auton Nerv Syst 1988;24:147-55.

13 Weissler AM, Leonard JJ, Warren JV. Effects of posture and atropine on the cardiac output. J Clin Invest 1957; 36:1656-62.

14 Chamberlain DA, Turner P, Sneddon JM. Effects of atropine on heart rate in healthy man. Lancet 1967;ii:12-5.

15 Katona PG, Jih F. Respiratory sinus arrhythmia: noninvasive measure of parasympathetic cardiac control J Appl Physiol 1975;39:801-5.

16 Pomeranz B, Macaulay RJB, Caudill MA, Kutz I, Adam D, Gordon D, et al. Assessment of autonomic function in humans by heart rate spectral analysis. Am J Physio 1985;248:H151-3.

17 Chess GF, Tam MK, Calaresu FR. Influence of cardiac neural inputs on rhythmic variations of heart period in the cat. Am J Physiol 1975;228:775-80.

18 Keele CA, Neil E, Joels N, eds. The heart rate. Samson Wright's applied physiology. 13th ed. New York: Oxford Wright's applied physiology. 13th

19 Eckberg DL. Temporal response patterns of the human sinus node to brief carotid baroreceptor stimuli. J Physiol sinus node to brief carotid

20 Gilbey MP, Jordan D, Richter DW, Spyer KM. Synaptic mechanisms involved in the inspiratory modulation of vagal cardio-inhibitory neurons in the cat. J Physio (Lond) 1984;356:65-78.

21 Burgos LG, Ebert TJ, Asiddao C, Turner LA, Pattison CZ Wang-Cheng $R$, et al. Increased intraoperative cardiovascular morbidity in diabetics with autonomic neuropathy. Anesthesiology 1989;70:591-7.

22 Weiner N. Atropine, scopolamine, and related antimuscarinic drugs. In: Goodman and Gilman's the pharmacological basis of therapeutics. 7th ed. London: Macmillan, 1985:130-44.

23 American Diabetes Association, American Academy of Neurology. Report and recommendations of the San Antonio conference and recommendations of the San 1988;11:592-7. 